\title{
Smartphone-Based VO2max Measurement With Heart Snapshot in Clinical and Real-world Settings With a Diverse Population: Validation Study
}

Dan E Webster ${ }^{1 *}, \mathrm{PhD}$; Meghasyam Tummalacherla ${ }^{1 *}$, MS; Michael Higgins ${ }^{2}$, MS, XT; David Wing ${ }^{2}$, CPT, MS, CBDT; Euan Ashley ${ }^{3}$, MD, PhD; Valerie E Kelly ${ }^{4}$ PT, PhD; Michael V McConnell ${ }^{5,6}$, MD, MSEE; Evan D Muse ${ }^{7}$, MD; Jeffrey E Olgin ${ }^{8}, \mathrm{MD}$; Lara M Mangravite ${ }^{1}, \mathrm{PhD}$; Job Godino ${ }^{2,7}, \mathrm{PhD}$; Michael R Kellen ${ }^{1 *}$, PhD; Larsson Omberg $^{1 *}, \mathrm{PhD}$

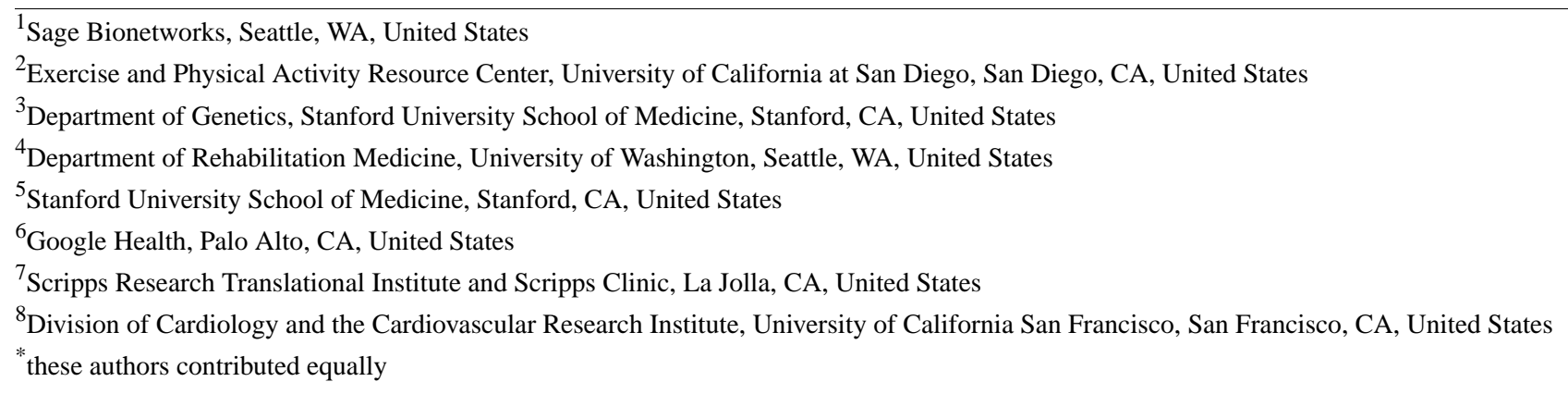

Corresponding Author:

Larsson Omberg, PhD

Sage Bionetworks

2901 3rd Ave \#330

Seattle, WA

United States

Phone: 12069288250

Email: 1arsson.omberg@sagebionetworks.org

\section{Abstract}

Background: Maximal oxygen consumption $\left(\mathrm{VO}_{2} \mathrm{max}\right)$ is one of the most predictive biometrics for cardiovascular health and overall mortality. However, $\mathrm{VO}_{2} \mathrm{max}$ is rarely measured in large-scale research studies or routine clinical care because of the high cost, participant burden, and requirement for specialized equipment and staff.

Objective: To overcome the limitations of clinical $\mathrm{VO}_{2} \mathrm{max}$ measurement, we aim to develop a digital $\mathrm{VO}_{2}$ max estimation protocol that can be self-administered remotely using only the sensors within a smartphone. We also aim to validate this measure within a broadly representative population across a spectrum of smartphone devices.

Methods: Two smartphone-based $\mathrm{VO}_{2}$ max estimation protocols were developed: a 12-minute run test (12-MRT) based on distance measured by GPS and a 3-minute step test (3-MST) based on heart rate recovery measured by a camera. In a 101-person cohort, balanced across age deciles and sex, participants completed a gold standard treadmill-based $\mathrm{VO}_{2}$ max measurement, two silver standard clinical protocols, and the smartphone-based 12-MRT and 3-MST protocols in the clinic and at home. In a separate 120-participant cohort, the video-based heart rate measurement underlying the 3-MST was measured for accuracy in individuals across the spectrum skin tones while using 8 different smartphones ranging in cost from US \$99 to US \$999.

Results: When compared with gold standard $\mathrm{VO}_{2}$ max testing, Lin concordance was $p_{\mathrm{c}}=0.66$ for $12-\mathrm{MRT}$ and $p_{\mathrm{c}}=0.61$ for 3-MST. However, in remote settings, the 12-MRT was significantly less concordant with the gold standard $\left(p_{\mathrm{c}}=0.25\right)$ compared with the 3-MST $\left(p_{\mathrm{c}}=0.61\right)$, although both had high test-retest reliability (12-MRT intraclass correlation coefficient=0.88; 3-MST intraclass correlation coefficient=0.86). On the basis of the finding that 3-MST concordance was generalizable to remote settings whereas 12-MRT was not, the video-based heart rate measure within the 3-MST was selected for further investigation. Heart rate 
measurements in any of the combinations of the six Fitzpatrick skin tones and 8 smartphones resulted in a concordance of $p_{\mathrm{c}} \geq 0.81$. Performance did not correlate with device cost, with all phones selling under US $\$ 200$ performing better than $p_{\mathrm{c}}>0.92$.

Conclusions: These findings demonstrate the importance of validating mobile health measures in the real world across a diverse cohort and spectrum of hardware. The 3-MST protocol, termed as heart snapshot, measured $\mathrm{VO}_{2}$ max with similar accuracy to supervised in-clinic tests such as the Tecumseh $\left(p_{\mathrm{c}}=0.94\right)$ protocol, while also generalizing to remote and unsupervised measurements. Heart snapshot measurements demonstrated fidelity across demographic variation in age and sex, across diverse skin pigmentation, and between various iOS and Android phone configurations. This software is freely available for all validation data and analysis code.

(JMIR Mhealth Uhealth 2021;9(6):e26006) doi: $10.2196 / 26006$

\section{KEYWORDS}

VO2max; heart rate; digital health; real-world data; cardiorespiratory fitness; remote monitoring; mobile phone; smartphone; validation

\section{Introduction}

\section{Background}

Expanding access to precision medicine will increasingly require patient biometrics to be measured in remote care settings. Traditionally, cardiovascular health has been assessed using risk scores such as the Framingham Risk Score [1], Reynolds Risk Score [2], Qrisk [3], and others, which integrate multiple factors including demographic data, comorbidities, and biometrics paired with imaging-based assessments measuring vascular blockage and blood flow in higher-risk and symptomatic individuals. Although these factors have a clear correlation with cardiovascular health, their inclusion in integrative risk calculations was promoted in part because they can be rapidly evaluated across many individuals. However, one of the most predictive biometrics for cardiovascular health [4] and overall mortality [5], maximal oxygen consumption $\left(\mathrm{VO}_{2} \mathrm{max}\right)$, is typically not incorporated in these risk calculators because of the high cost, participant burden, and specialized equipment and staff needed to obtain this measurement $[6,7]$.

Cardiorespiratory fitness, as measured by $\mathrm{VO}_{2}$ max, represents the integrated function of physiological systems involved in transporting oxygen from the atmosphere to the skeletal muscles to perform physical work. Existing gold standard techniques for measuring $\mathrm{VO}_{2}$ max are based on protocols that use exercise on a treadmill or stationary bicycle paired with the direct measurement of oxygen consumption at various workloads, including maximal exertion $[8,9]$. However, the requirement to exercise at the maximal aerobic threshold limits deployment in some populations for safety reasons, and the need for specialized equipment and personnel has prohibited widespread adoption of $\mathrm{VO}_{2}$ max testing in research and clinical settings.

\section{Objectives}

Limitations of gold standard $\mathrm{VO}_{2} \max$ measurements have led to the development of numerous "silver standard" [10] $\mathrm{VO}_{2} \max$ estimation protocols that rely on simpler equipment or submaximal levels of exertion. These protocols trade off measurement accuracy for ease of deployment in a wider range of settings and for populations with differing levels of capacity [11]. However, these protocols were typically developed and validated in small, homogeneous populations, and some subsequent validation studies have been criticized for demonstrating participant selection bias [12]. To overcome these limitations, we aim to develop a digital $\mathrm{VO}_{2}$ max estimation protocol that could be self-administered remotely using only the sensors within a smartphone, and we also aim to validate this measure within a broadly representative population. Previous efforts have used a smartphone-based approach to measure $\mathrm{VO}_{2} \max$, but these validation studies are rarely conducted outside of clinical settings [13]. Therefore, we aim to validate our measurements in remote, unsupervised real-world settings.

\section{Methods}

\section{Development of Smartphone Sensor-Based Measurements of $\mathrm{VO}_{2}$ max}

Two silver standard $\mathrm{VO}_{2}$ max estimation protocols were chosen as the basis for developing the smartphone tests. The first is the Cooper protocol [14], comprising a 12-minute run test (12-MRT), where individuals cover as much distance as possible in 12 minutes on a flat course. The Cooper protocol estimates $\mathrm{VO}_{2}$ max from the total distance traveled during the 12 minutes. The other is the Tecumseh protocol [15], which comprises a 3-minute step test (3-MST), where individuals step up and down an 8-inch step at a constant rate for 3 minutes. In the 3-MST protocol, $\mathrm{VO}_{2} \max$ was estimated from the heart rate measurements during the recovery period. In adopting these protocols for smartphones, we developed self-guided instructions with GPS to record distance during the 12-MRT and a smartphone camera to record heart rate during recovery for the 3-MST (Multimedia Appendix 1).

\section{$\mathrm{VO}_{2} \max$ Validation Cohort Procedures and Measures}

All study procedures were approved by the University of California, San Diego (UCSD) Institutional Review Board (approval number 171815). All participants provided written informed consent and attended two in-person study visits at the Exercise and Physical Activity Resource Center (EPARC).

A convenience sample of 101 adults aged between 20 and 79 years was recruited, largely balanced across age deciles and sex (Multimedia Appendix 2). Potential participants were contacted by trained EPARC staff via email or telephone and they 
underwent a screening to ascertain their eligibility. Participants were included if they were (1) able to consent and participate in the study using English; (2) aged between 20 and 79 years; (3) willing and able to attend two in-person study visits that included either a $\mathrm{VO}_{2}$ max test or a 12-MRT and a 3-MST within a 2-week period; (4) willing and able to undertake up to three 12-MRT and 3-MST at home over a 2-week period; (5) willing and able to download the smartphone app developed to measure cardiorespiratory fitness on a compatible Android or iOS device and use it during all tests over a 2 -week period; and (6) willing and able to download the Fitbit smartphone app on a compatible Android or iOS device and connect and wear a study-provided Fitbit Charge 2 during all tests over a 2-week period. Participants were excluded if they (1) were >12 weeks pregnant; (2) had a heart or cardiovascular condition, including coronary artery disease, congestive heart failure, diagnosed abnormality of heart rhythm, atrial fibrillation, and/or a history of myocardial infarction; (3) required the use of an external device to assist heart rhythm (eg, a pacemaker); (4) had a serious respiratory disease, including chronic obstructive pulmonary disease, exercise-induced asthma, and/or pulmonary high blood pressure; (5) required use of supplemental oxygen; (6) required use of a beta-blocker or other medications known to alter heart rate; and (7) answered "yes" to one or more questions in the American College of Sports Medicine's Physical Activity Readiness Questionnaire and/or reported two or more risk factors for exercise testing and did not receive subsequent medical clearance. The Physical Activity Readiness Questionnaire is a widely accepted tool used to assess an individual's fitness for tests involving cardiovascular exercise [16].

Upon completion of the telephone screening (and, if necessary, receipt of medical clearance), potential participants were scheduled to attend the first testing session at the UCSD. They were asked to report to the testing session well hydrated and in an athletic attire. Participants were guided through the process of downloading and installing the smartphone app developed to measure cardiorespiratory fitness, as well as the Fitbit smartphone app, and they were fitted with a wrist-worn Fitbit Charge 2 according to the manufacturer's recommendations. Participants were asked to provide their age, sex at birth, ethnicity, and race. Weight (to the nearest $0.1 \mathrm{~kg}$ ) and height (to the nearest $0.1 \mathrm{~cm}$ ) were measured using a calibrated digital scale and stadiometer (Seca 703, Seca GmbH \& Co. KG). Both weight and height were measured with participants wearing lightweight clothes without shoes, and two separate measurements were averaged (if weight or height measurements differed by more than $1 \%$, then a third measure was taken, and the average of the two measures that differed by less than 0.2 $\mathrm{kg}$ or $0.5 \mathrm{~cm}$, respectively, was used).

At the first testing session, participants either undertook a $\mathrm{VO}_{2}$ max test or an in-clinic 3-MST and 12-MRT. A randomization procedure implemented before the scheduling of the first testing session determined which test procedure participants undertook during the first testing session. The participants were then expected to complete the other test procedures during the second testing session.

\section{Treadmill-Based Gold Standard $\mathrm{VO}_{2}$ max Measurement}

Participants completed a maximal graded exercise test on a Woodway 4Front treadmill (Woodway) calibrated monthly for accuracy of speed and grade. The maximal graded exercise test protocol began with a warm-up at a self-selected pace on a treadmill for 5 to 10 minutes. During the warm-up, EPARC staff explained how to use the Borg Rating of Perceived Exertion scale and reminded participants that they were expected to achieve their maximal level of exertion [17].

The participants were then equipped with a breath mask that covered the nose and mouth (KORR Medical Technologies) and a Bluetooth-enabled heart rate monitor worn on the chest (Garmin). The preprogrammed treadmill protocol began with the participants running at $5 \mathrm{mph}$ with a $0 \%$ incline for 3 minutes. The workload was then increased by approximately 0.75 , which is the metabolic equivalent of tasks every minute. This was achieved via an increase in speed $(0.5 \mathrm{mph}$ per min) each minute until the participant was $0.5 \mathrm{mph}$ above their self-determined comfortable speed or until a maximal speed of $9 \mathrm{mph}$ was reached. If the participant's capacity allowed them to continue beyond this upper speed limit before reaching volitional fatigue, then the treadmill speed was kept constant, but the grade (ie, incline) of the treadmill was increased by $1 \%$ each minute until volitional fatigue was reached. The Borg Rating of Perceived Exertion scale was assessed during the final 10 seconds of each minute, and the protocol continued until the participant signaled to stop (ie, indication of volitional fatigue). Upon indication of volitional fatigue, the treadmill was immediately slowed to $2 \mathrm{mph}$, and participants were encouraged to walk until completely recovered. Breath-by-breath oxygen uptake $\left(\mathrm{VO}_{2}\right)$ was continuously measured using an indirect calorimeter (COSMED) that was calibrated for gas volume and fractional composition immediately (ie, $<30 \mathrm{~min}$ ) before the start of the maximal graded exercise test protocol.

\section{Tecumseh Test (3-MST) and Cooper Test (12-MRT) In-Clinic Procedure}

All participants were fitted with a chest-worn heart rate monitor (Polar) that was used for real-time monitoring by trained EPARC staff throughout both the 12-MRT and 3-MST. For the 3-MST, participants were instructed to step up and down from a single step 8 inches in height at a rate of 24 steps per minute for 3 minutes [18]. The cadence of stepping was monitored by trained EPARC staff. The radial pulse was measured from the $31 \mathrm{st}$ second to the 60th second after 3 minutes of stepping. Upon completion of the test, the participants were asked to sit in a chair and rest. After a minimum of 10 minutes of rest, participants completed a 5-minute self-determined light intensity warm-up. They were then instructed to cover as much distance as possible on a flat 400-m track for 12 minutes. The distance traveled was measured after 12 minutes [14].

\section{Distance Estimation Using Privacy-Preserving GPS Data}

The distance recorded by the smartphone during the 12-MRT was validated against the actual distance. The smartphone recorded displacement information sampled at $1 \mathrm{~Hz}$, which 
consists of relative location measurements, that is, the change in location with regard to the last recorded measurement. The iPhones (Apple Inc) measured displacement in meters whereas the Android smartphones measured relative changes in latitude and longitude, requiring an estimate of the absolute latitude and longitude to be added back into the measurements to obtain an accurate estimate of distance.

The first distance estimation method entailed summing the Euclidean distances between subsequent GPS points. As GPS measurements have a range error dependent on atmospheric effects and numerical errors, a second method was used to compute the distance after smoothing the trajectory of the GPS path using a Savitzky-Golay smoothing filter.

\section{Camera-Based Heart Rate Estimation}

Blood flow through the fingertip was measured through video with a rear-facing camera while the flash was on. The resting heart rate was captured with 20 seconds of recording, whereas the 3-MST required 60 seconds of recording. During the capture, we found it was important to fix the focal length to infinity, turn off any high dynamic range settings (if applicable), and set the frame rate to $60 \mathrm{~Hz}$ if possible, and if not, the default highest allowed by the phone. We did not record the video in order to preserve privacy associated with the inadvertent capture of identifiable objects in the frame before covering the lens with the finger, but instead summarized each video frame to the mean of all pixel intensities per color channel in the red, green, blue space.

These intensities yielded three time series, one for each color. These time series were filtered and mean-centered before being split into shorter 10-second windows. By assuming a periodic signal for these windows, the autocorrelation function (ACF) was used to estimate the period by finding the peaks and their corresponding lags. The relative magnitude of the peaks to the maxima of the ACF was used to generate a confidence score, which quantifies the extent to which the signal is periodic or if the peak at the fundamental frequency (ie, the peak with the highest magnitude) is a spurious peak. The ACF is calculated over a 10 -second window, as this provides sufficient heart beat observations postprocessing to estimate heart rates ranging from 45 to $210 \mathrm{bpm}$.

To filter potentially spurious peaks, a magnitude threshold relative to the magnitude of the peak at zero lag was used. The confidence score was calculated as the ratio of the magnitude of the peak corresponding to the fundamental frequency to the next peak. The confidence score is an indicator of the periodicity of the signal, a property indicative of the heart rate signal in a short finite time window. The different color channels were merged by choosing the heart rate estimate from the channel (red or green) that had the maximum confidence score within a given window.

\section{Estimation of $\mathrm{VO}_{2} \max$}

\section{3-Minute Step Test}

Multiple formulas for predicting $\mathrm{VO}_{2}$ max from the Tecumseh step test and its variations have been developed [15]; here, we used the following established by Milligan [19]:

$$
\mathrm{VO}_{2} \max =\frac{d_{12}-504.9}{44.73}(\mathrm{ml} / \mathrm{kg} / \mathrm{min})
$$

where HB3060 is the number of beats between 30 and 60 seconds after the step test, age is the age of the subject, and sex is 0 if male and 1 if female.

\section{2-Minute Run Test}

$\mathrm{VO}_{2} \mathrm{max}$ for the 12-MRT is estimated from the following formula, where $d_{12}$ is the distance covered in meters [14]:

$V \mathrm{O}_{2} \max =83.477-0.586(\mathrm{HB} 30$ to60 $)-0.404($ age $)-7.030(\mathrm{sex})(\mathrm{ml} / \mathrm{kg} / \mathrm{min})$

\section{Heart Rate Calibration Study Procedures and Measures}

All study procedures were approved by the UCSD Institutional Review Board (approval number 181820). All participants provided written informed consent and attended one in-person study visit at the EPARC.

A convenience sample of 120 adults, aged 18-65 years, of six different skin types were asked to participate in this study. We aimed to recruit an equal ratio of male and female participants, as well as an equal number of participants with each skin type, as determined by the Fitzpatrick scale. Participants were included if they were (1) able to consent and participate in the study in English and (2) aged between 18 and 65 years. Participants were excluded if they had (1) peripheral neuropathy or (2) tattoos or scarring at the measurement site (index finger and/or wrist). Potential participants were contacted by trained EPARC staff via email or telephone, and they were asked to complete the screening to ascertain their eligibility.

To establish the Fitzpatrick skin type of the cohort during recruitment, participants were asked to self-assess their Fitzpatrick skin type based on visual comparison with images of well-known celebrities with diverse pigmentation levels. As self-assessment of skin type can have variable accuracy [20,21], spectrocolorimetry was also used as an objective standard [22]. Spectrocolorimetry measurements were performed on the underside of the jaw using Pantone RM200QC. To calculate pigmentation in the individual typology angle color space, the $\mathrm{L}^{*}$ and $\mathrm{b}^{*}$ parameters from the spectrocolorimetry measurements were used according to the formula:

$$
\begin{aligned}
& \text { individual typology angle }=\left[\arctan \left(\left(\mathrm{L}^{*}-50\right) / \mathrm{b}^{*}\right)\right] \times \\
& 180 / 3.14159 \text { (1) }
\end{aligned}
$$

Using this formula, skin color types can be classified into six groups, ranging from very light to dark skin: very light $>55^{\circ}>$ light $>41^{\circ}>$ intermediate $>28^{\circ}>\tan >10^{\circ}>$ brown $>-30^{\circ}>$ dark [22].

Upon completion of the telephone screening, potential participants were scheduled to attend the first testing session at the UCSD. Participants were asked to provide their age, sex at birth, ethnicity, and race. All participants were fitted with a chest-worn heart rate monitor that was used for real-time monitoring by trained EPARC staff throughout testing. Heart rate was also monitored using a finger-based pulse oximeter (Nonin Medical, Inc). The finger-based pulse oximeter was attached to the participants' index finger, and the time was 
synchronized between the computer and the device. Trained research staff visually confirmed that the photoplethysmograph was reading accurately before starting measurements on smartphone devices.

Participants were then given the first of 8 smartphones: Huawei Mate SE, LG Stylo 4, Moto G6 Play, Samsung Galaxy J7, Samsung Galaxy S9+, iPhone8+, iPhoneSE, and iPhoneXS. They were instructed by trained research staff to stand still and gently cover the camera and flash on the back of the smartphone with their fingertip, as their heart rate was captured by our preloaded smartphone app. The time on the Polar app was recorded at the time the measurement began on the smartphone app. Measurements with each smartphone lasted 60 seconds in duration. Processed data from the finger-based pulse oximeter were parsed and transformed with custom scripts to generate continuous photoplethysmography data in a format suitable for comparison with the heart rates from the phones.

\section{Statistical Analysis}

Demographic data were described using univariate summary statistics (eg, proportions, means, and SDs). Test validity for heart rate estimates and $\mathrm{VO}_{2} \max$ was visualized using Bland-Altman plots [23] and compared using the Lin concordance index [24]. The heart rate errors were also compared using percent error. Analyses were performed in both $\mathrm{R}$ and Python.

\section{iOS and Android Heart Snapshot Software Modules}

The code for the heart snapshot modules and sample Android [25] and iOS [26] apps are available under an open-source license.

\section{Results}

\section{Validation in a Clinical Setting}

To assess the validity of the 3-MST and 12-MRT smartphone measurements, gold standard $\mathrm{VO}_{2}$ max treadmill testing was performed with 101 participants distributed across age deciles 20-80 years. Every participant also performed the silver standard and smartphone 12-MRT and 3-MST protocols in the clinic, with three instances of each smartphone protocol performed over 2 weeks without supervision in the participant's home environment (Figure 1). 
Figure 1. Validation protocol and primary results of validation. (A) Participants in the study were randomized into two groups. The first group (denoted by the downward-facing arrow at top) performed a gold standard $\mathrm{VO}_{2}$ max protocol and received training on day 1 . The second group performed the two silver standard protocols concurrently with the smartphone protocols on day 1 (denoted by the upward-facing arrow at bottom). Both groups then performed the two smartphone protocols remotely up to three times during a 2-week period. (B) to (E) show Bland-Altman plots comparing the gold standard $\mathrm{VO}_{2}$ max with smartphone measures from: (B) 12-MRT performed in clinic, (C) 12-MRT performed remotely (up to 3 repeats per participant), (D) 3-MST in clinic, and (E) 3-MST remotely. $\mathrm{VO}_{2}$ max: maximal oxygen consumption; 3-MST: 3-minute step test; 12-MRT: 12-minute run test.

A

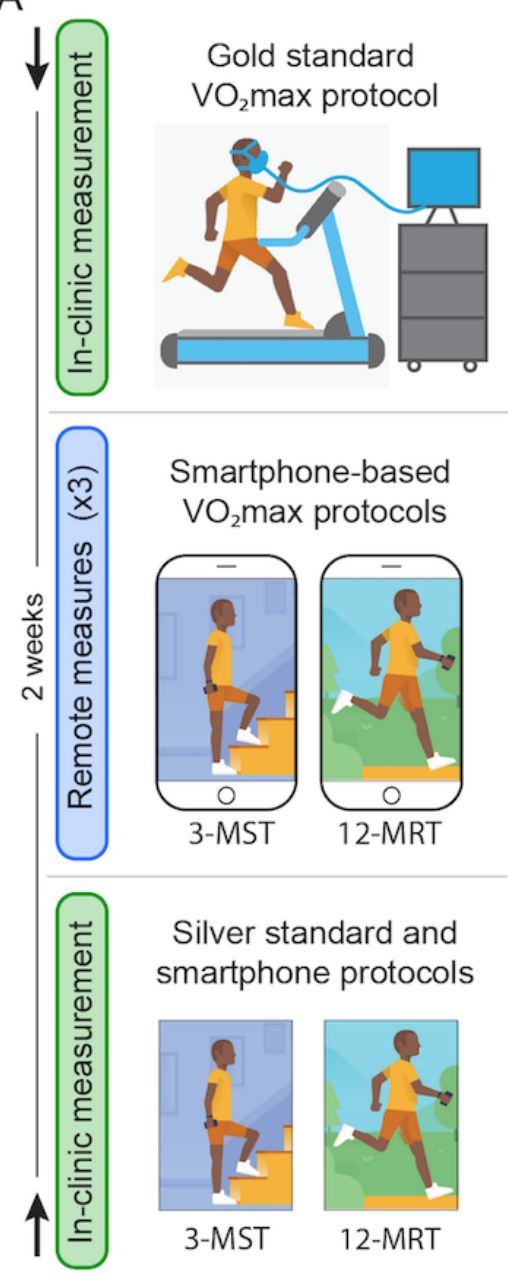

B

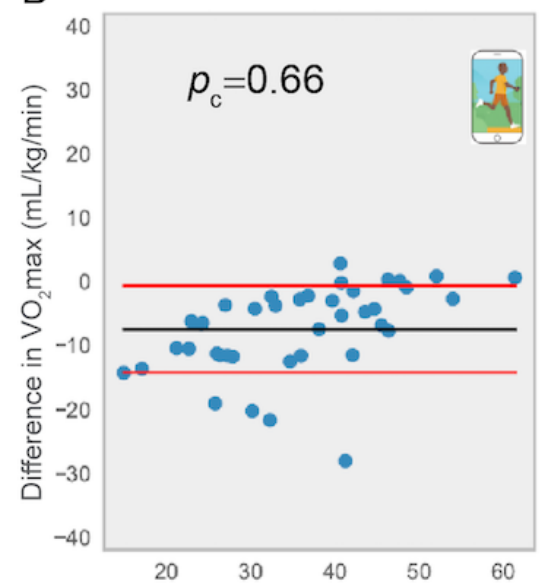

D

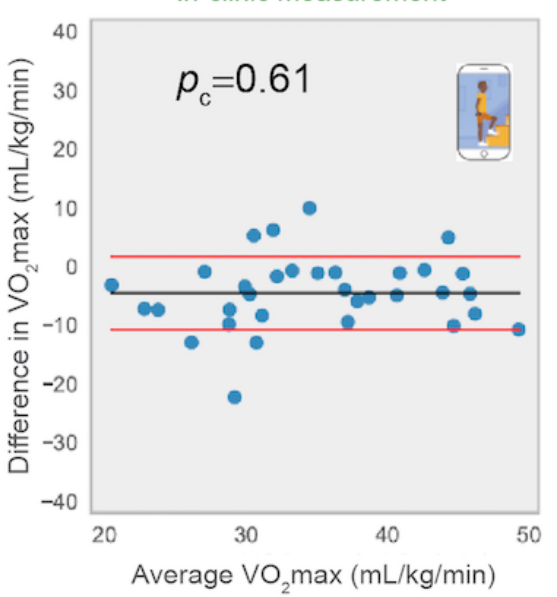

C

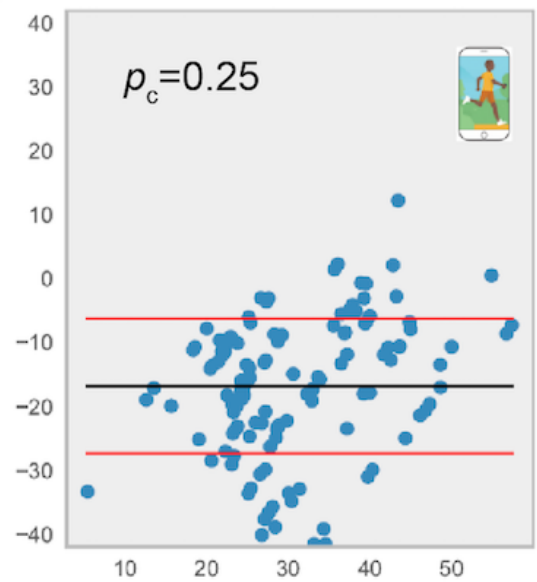

E

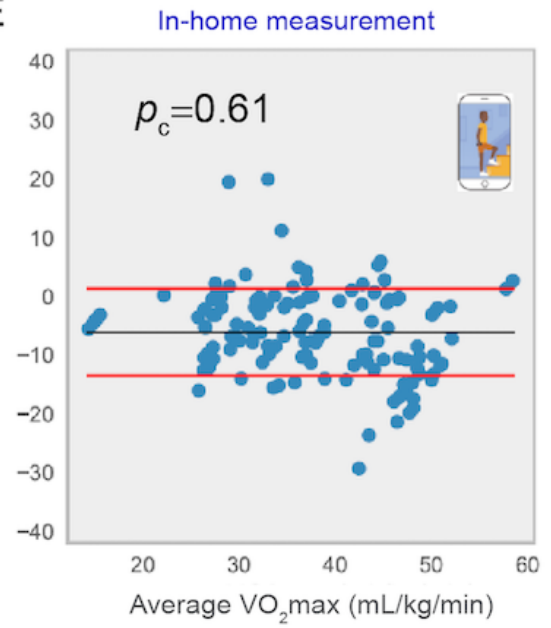

The in-clinic 12-MRT distance was measured on a 400-m track and by the smartphone GPS. The in-clinic heart rate was measured via radial pulse by trained research staff, a chest-worn Polar heart monitor, a wrist-worn Fitbit Charge 2, and a smartphone camera with the flash activated. Comparisons between the gold standard, silver standard, and smartphone-based protocols for $\mathrm{VO}_{2}$ max estimation were performed using Bland-Altman analysis [23] and the Lin concordance index $\left(p_{\mathrm{c}}\right)$. The concordance between gold standard $\mathrm{VO}_{2} \max$ and the silver standard Cooper protocol $\left(p_{\mathrm{c}}=0.61\right.$; Figure 2) and the silver standard Tecumseh protocol $\left(p_{\mathrm{c}}=0.70\right.$;
Figure 2) were in line with previously published results [27-29]. Concordance of smartphone-based protocols with gold standard $\mathrm{VO}_{2}$ max testing was $p_{\mathrm{c}}=0.66$ for the 12-MRT (Figure 1) and $p_{\mathrm{c}}=0.61$ for the 3-MST (Figure 1). The concordance of smartphone-based protocols with silver standard protocols was $p_{\mathrm{c}}=0.96$ for the 12-MRT and $p_{\mathrm{c}}=0.94$ for the 3-MST. These results demonstrate that the smartphone-based protocols fall short of recapitulating gold standard $\mathrm{VO}_{2}$ max testing but are highly concordant with validated silver standard $\mathrm{VO}_{2} \max$ estimation protocols in a laboratory setting. 
Figure 2. Comparison of in-clinic performance of silver standard protocols relative to the gold standard for (A) 12-minute run test (12-MRT) and (B) 3-minute step test. For each plot, we are showing the difference between the ground truth maximal oxygen consumption measurement and measurements obtained using the distance run around a track (for A) and heart rate via radial pulse measured by trained research staff (for B) as per Tecumseh protocol. This distance was also measured using GPS and heart rate was measured using a chest strap and Fitbit. The concordance between distance measured around the track and measured using the GPS in the phone was 0.96. (C) to (F) show the concordance of the 12-MRT test for different values of self-reported effort. $\mathrm{VO}_{2}$ max: maximal oxygen consumption.

A
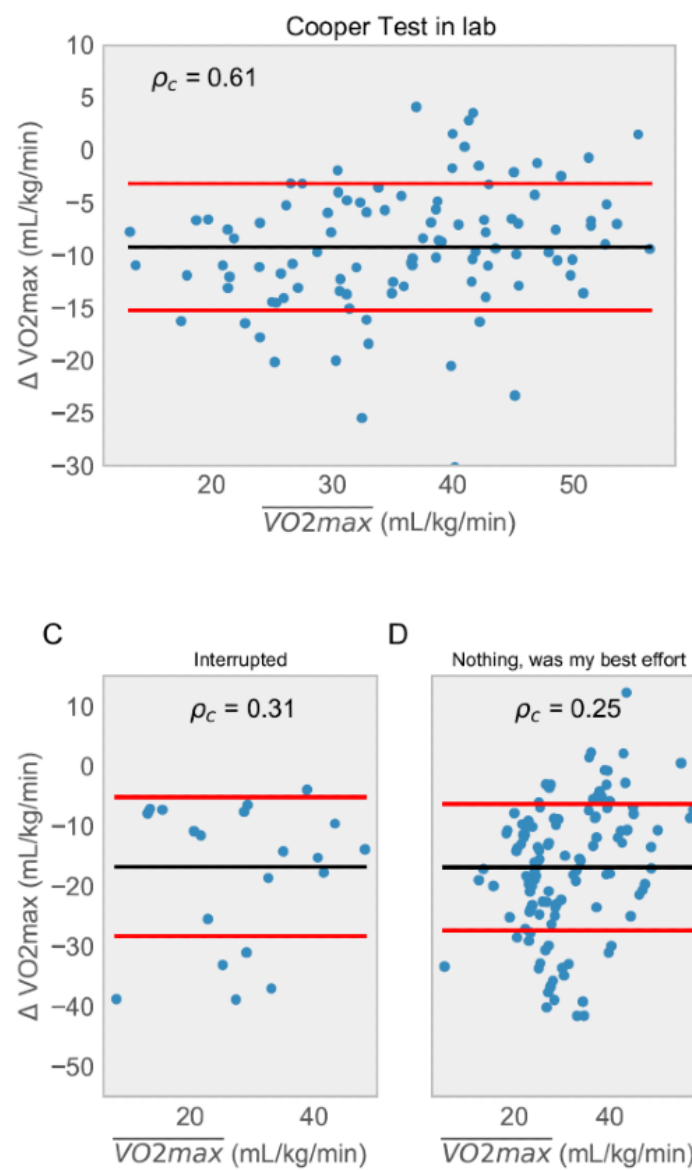

$\mathrm{D}$

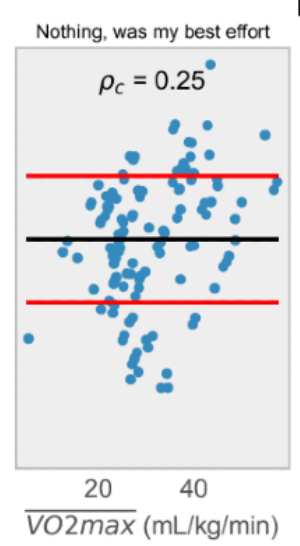

B

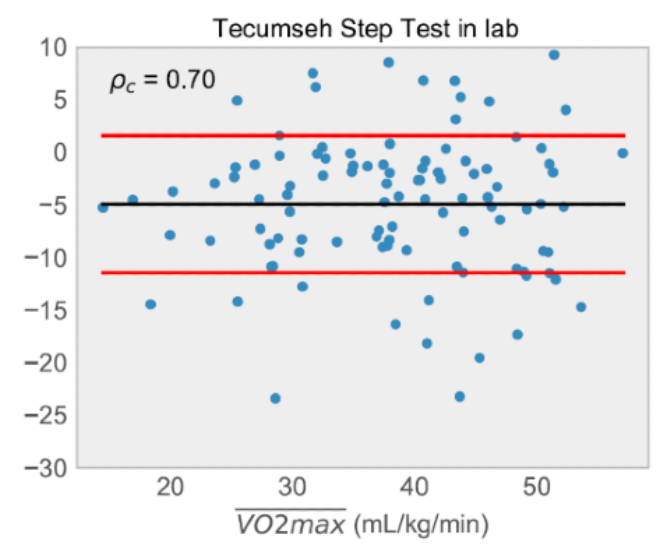

E
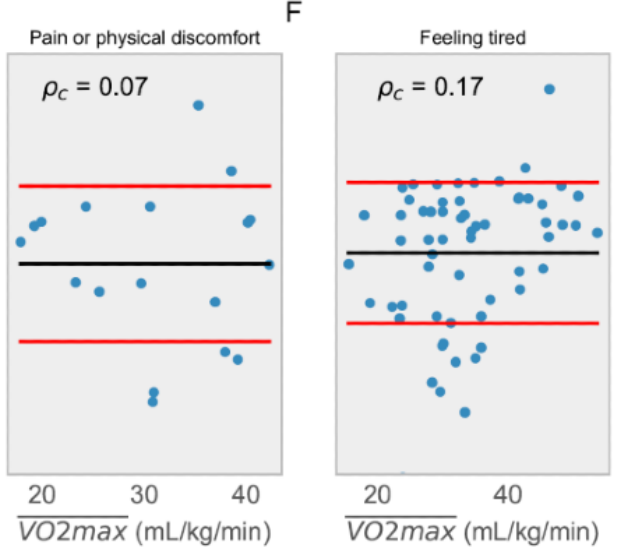

\section{Validation in a Remote Setting}

To investigate whether the concordance of in-clinic measurements would generalize to remote and unsupervised settings, the smartphone protocols were also performed up to three times at home by each participant. We observed an approximately equal test-retest reliability between the two tests (3-MST intraclass correlation coefficient $=0.86$; 12-MRT intraclass correlation coefficient $=0.88$ ). However, although the 3 -MST translated well to an unsupervised setting $\left(p_{\mathrm{c}}=0.61\right.$; Figure 1), the 12-MRT demonstrated a pronounced drop in concordance ( $p_{\mathrm{c}}=0.25$; Figure 1$)$, despite a highly accurate distance measurement from the smartphone $\left(p_{\mathrm{c}}=0.96\right)$ based on comparisons made in a clinical setting.

As the 12-MRT is dependent on maximal effort, participants were surveyed directly after their run about their performance. In $63.4 \%(137 / 216)$ of runs performed remotely, participants reported the run to be "their best effort." Therefore, only 137 runs were used to estimate $\mathrm{VO}_{2} \mathrm{max}$ in our analysis. Figure 2 captures the results of all 216 runs subdivided by self-reported effort. Although the context-dependent failure of the 12-MRT in remote settings may be attributable to many factors, this result highlights the importance of both clinical and unsupervised real-world evidence for the validation of novel digital health measurement modalities.

\section{Calibration of the Heart Snapshot Measurement for a Diverse Audience}

The smartphone-based 3-MST protocol, hereafter referred to as heart snapshot, was generalizable between clinical and remote assessments and was robust over a large range of fitness levels. Maintaining high concordance during unsupervised measurements is necessary to achieve the scale intended for the targeted 1 million participants in the All of Us Research Program (AoURP) [30], which will use a "bring-your-own-device" strategy for remote self-measurement of $\mathrm{VO}_{2}$ max. AoURP also aims to recruit a study population matching the full demographic diversity of the United States, emphasizing the inclusion of groups often underrepresented in biomedical research, such as ethnic and racial minorities. As prior studies have shown differing results as to whether optical techniques for heart rate detection (photoplethysmography) can be demographically biased [31,32], we aimed to investigate any differences in heart snapshot accuracy across variations in skin tone. A follow-up calibration study for heart rate measurements was conducted 
with 120 participants distributed approximately evenly across defined Fitzpatrick skin types [33], using 8 different smartphones ( 3 iPhones and 5 Android smartphones ranging in cost from US \$99 to US \$999 at the time of writing). These phones were chosen to be representative of different operating systems, quality of sensors, processing speed, and camera configuration. Importantly, we observed no significant difference in heart rate measurement accuracy between categorical Fitzpatrick skin types or systematic measurement error proportional to skin color at either end of the spectrum (Figure 3).

Figure 3. Validation of heart rate measurements across different skin tones and hardware configurations in the calibration study. (A) Percent error in heart rate estimation from ground truth as a function of different colors captured by spectrocolorimetry under the jaw. Each dot represents a 10 second window of heart rate in one individual. (B) Distribution of concordance between heart rate using pulse oximetry and smartphone as the confidence cutoff is changed. Red line represents the chosen cutoff used for analysis. (C) Concordance as a function of smartphone models and Fitzpatrick skin tones. ITA: individual typology angle.

$$
\text { A }
$$

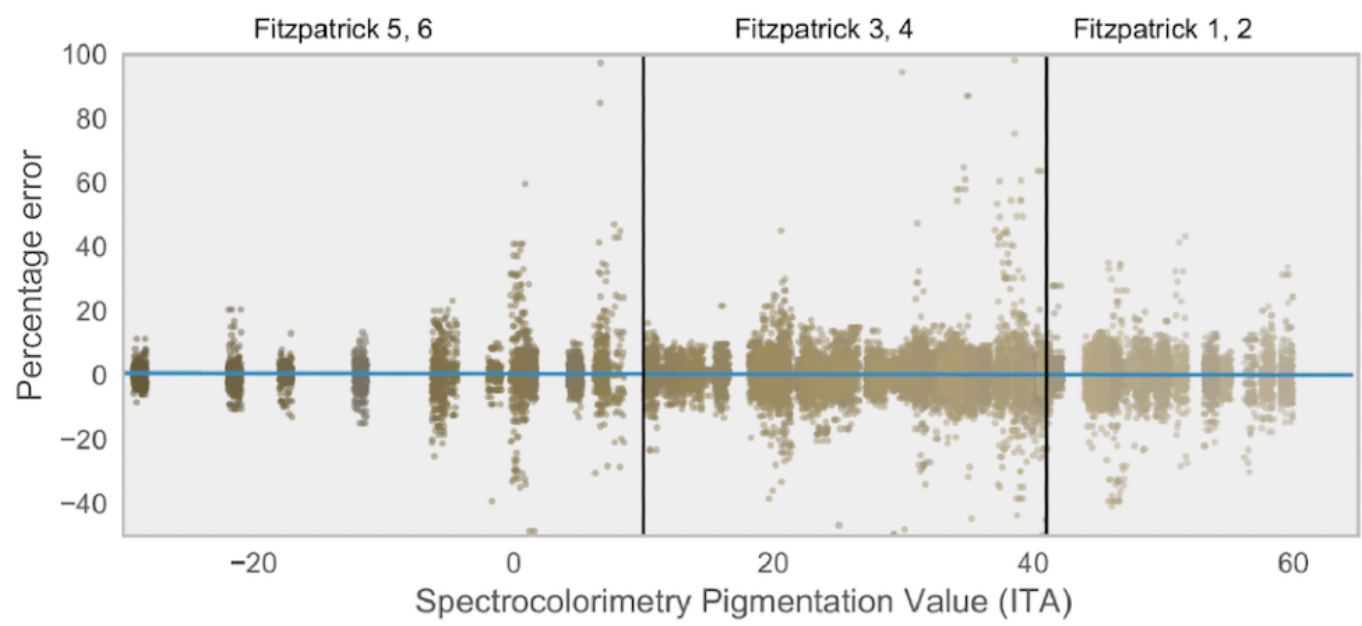

B

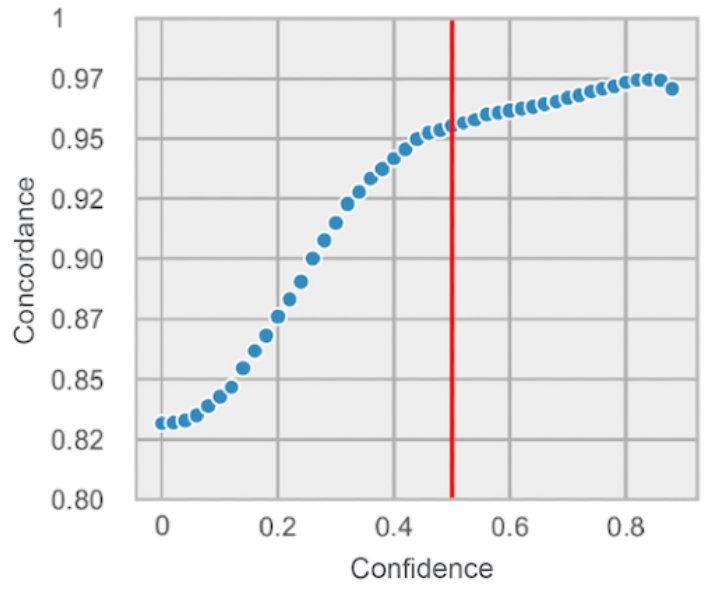

\section{Internal Quality Control Procedures for Heart Snapshot}

To facilitate quality control of the measurements across different smartphones, a confidence score was developed to provide a readout of the quality of the heart rate measurements. This confidence score is derived from the ACF of the heart rate signal across 10 -second measurement windows. Using the calibration study results, a balance between the quality of measurements was weighed against the loss of data by choosing a filtration cutoff at a confidence level of $\geq 0.5$. This resulted in a $p_{\mathrm{c}}=0.95$,

\section{C}

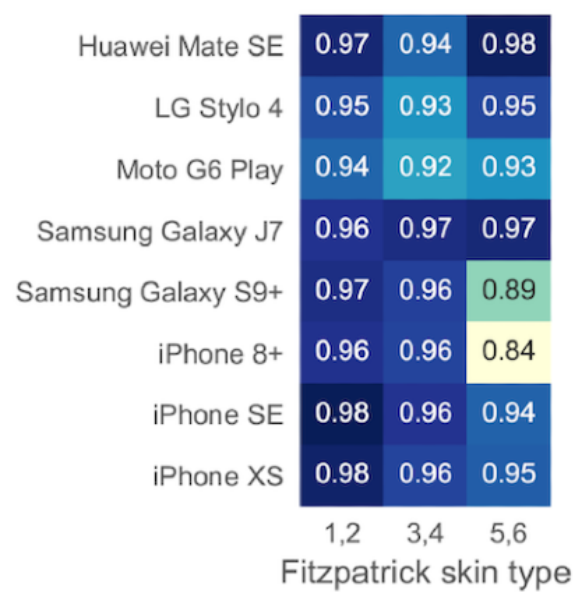

in the calibration cohort between a pulse oximetry pulse measurement and the camera-estimated heart rate (Figure 3). In selecting this confidence score as a cutoff, we observed that $80.41 \%(28,032 / 34,859)$ of all measurement windows were retained in this calibration cohort (Figure 4). The same cutoff was used in the validation of heart snapshot against gold standard $\mathrm{VO}_{2} \max$, where the heart rate concordance with a chest-worn Polar heart monitor was $p_{\mathrm{c}}=0.95$ and $p_{\mathrm{c}}=0.83$ when compared with a wrist-worn Fitbit Charge 2, both at home and in the clinic. This can be compared with $p_{\mathrm{c}}=0.92$ between Polar and Fitbit Charge 2 (Figure 5). 
Figure 4. Effect of different confidence cutoff on the amount of missing data from the calibration study. (A) Distribution of best confidence across red and green channels in the calibrations study and (B) percent of the 10 second windows that are filtered out at different cutoffs of the confidence score. The cutoff used in the analysis is 0.5 marked by the red line.

A

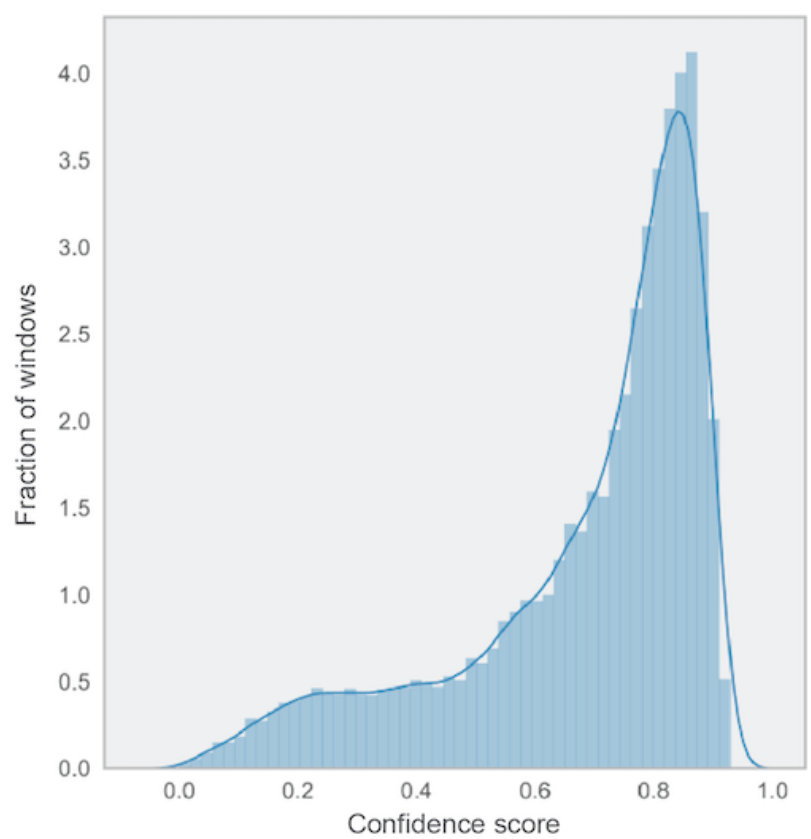

B

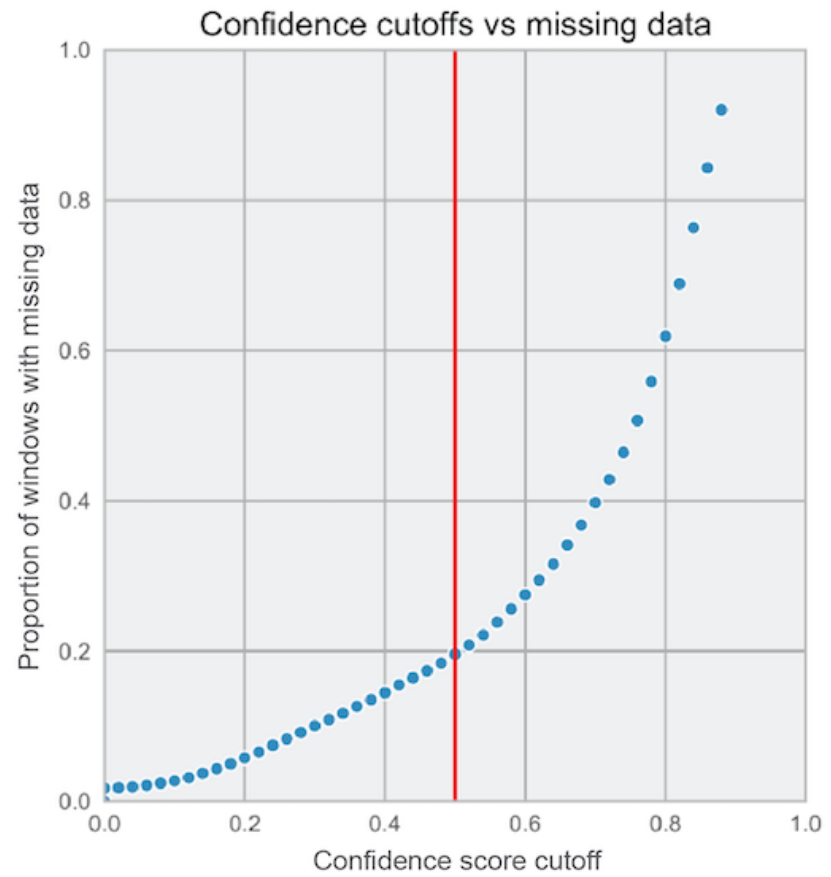

Figure 5. Bland-Altman analysis comparing heart rate measurements in the validation study using data collected during the Tecumseh tests. In the validation cohort, participants used multiple ways of collecting heart rate. The method being tested, the smartphone camera, was compared to: (A) a Polar chest strap (considered a gold standard) while in the clinic when both were used and (B) a Fitbit worn during the entirety of the study. (C) We also compared the Polar strap to the Fitbit for all time that both were worn. HR: heart rate.

A

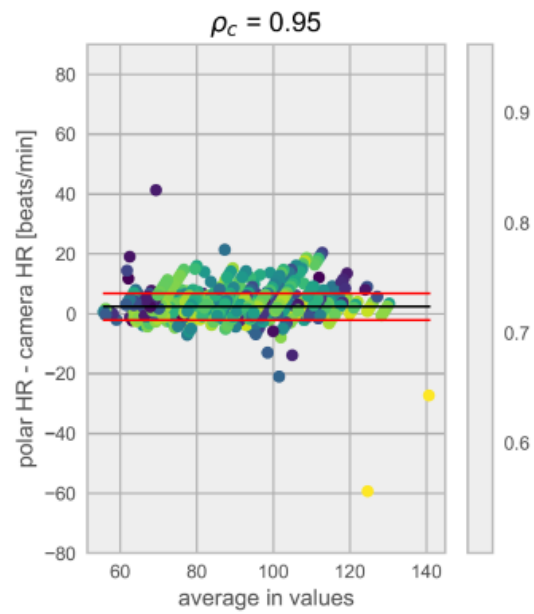

B

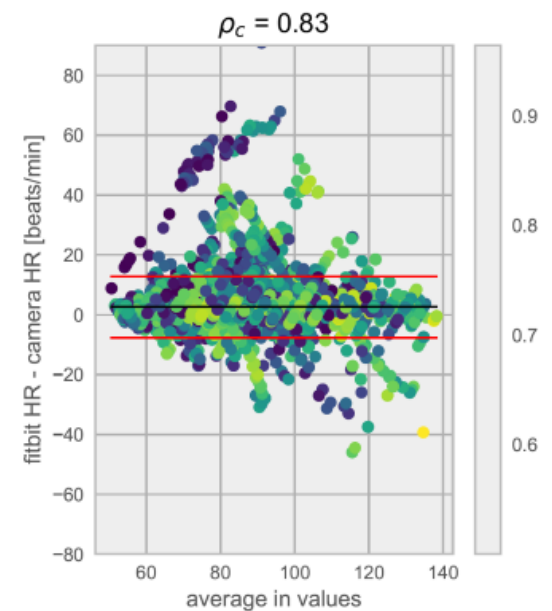

C

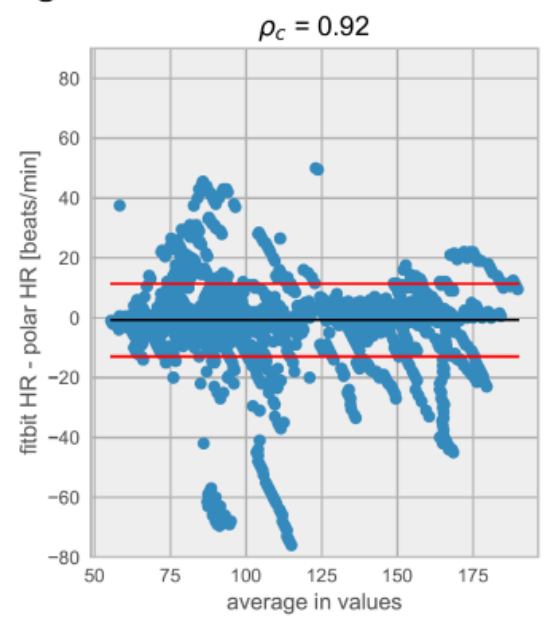

Taken together, heart snapshot heart rate measurements in any of the combinations of the Fitzpatrick skin tones and 8 smartphones used in the calibration study resulted in a concordance greater than or equal to $p_{\mathrm{c}}=0.84$ (Figure 3 ), which is in line with previous smartphone-based modalities for heart rate monitoring [34]. Importantly, performance did not correlate with device cost, with all phones selling for under US \$200 performing better than $p_{\mathrm{c}}>0.92$ for any skin tone.

\section{Discussion}

\section{Principal Findings}

In summary, heart snapshot measured $\mathrm{VO}_{2}$ max with similar accuracy to supervised, in-clinic tests such as the Tecumseh or Cooper protocols, while also generalizing to remote and unsupervised measurements. Heart snapshot measurements demonstrated fidelity across demographic variation in age and sex, across diverse skin pigmentation, and between various iOS and Android phone configurations.

The results from our validation study performed in unsupervised, remote environments showed that heart snapshot, which is based 
on a 3-MST protocol, generalized to real-world settings but the 12-MRT protocol did not. Although it is difficult to definitively determine the reason for the poor concordance of 12-MRT, we suspect that this might be attributed to the Hawthorne effect, where people perform better when they are under constant observation at a track. It could also be purely environmental, where traffic, hills, and distractions impede uninterrupted running. This indicates the importance of testing and validating digital health measures in a representative setting.

\section{Limitations}

An important limitation of this study is that we did not include any individuals in our cohort with a known irregular heart rhythm, so we cannot extend our claims of validity to that population. Similarly, although we made efforts to include individuals across different age deciles, we focused solely on adults (aged over 18 years) and our age decile from 60 to 70 years did not include participants older than 65 years. This work was limited to the biometrics of resting heart rate and $\mathrm{VO}_{2} \mathrm{max}$, but using the same technology could also be extended to measure heart rate recovery in minute intervals after exertion, which would provide a valuable biometric that has been associated with prediction of overall mortality [30]. Heart snapshot attempts to maximize concordance with gold standard methods for estimating $\mathrm{VO}_{2} \mathrm{max}$, but it is worth noting that this analysis used an existing validated algorithm [27] that was based on in-clinic procedures and measurement tools. Heart snapshot could become more personalized than traditional protocols, for example, adapting to a participant's maximum step cadence as measured by smartphone accelerometry. Further concordance with gold standard measures may be achieved by optimizing the parameters of the traditional algorithm or including new variables, but this will require a distinct cohort to test any models that have been trained on this data set.

\section{Comparison With Prior Work}

Although multiple devices can estimate $\mathrm{VO}_{2} \mathrm{max}$, including several currently marketed consumer devices [35], the underlying data and algorithms are usually not published. The lack of data and method transparency limits the utility of these approaches for discovery-based research, where reproducibility is paramount. In contrast, an open approach to method validation can also serve as a foundation for downstream research in different conditions or populations to generate normative data for interpreting results [36].

As many dedicated hardware devices for digital health in the consumer sphere have experienced short half-lives of availability, we believe that the dependency only on a smartphone with a flash and camera may provide a greater degree of future-proofing for heart snapshot. This will be important for consistent, longitudinal measurements that may uncover patterns of $\mathrm{VO}_{2} \mathrm{max}$ variance over time, especially in large-scale studies such as the AoURP.

\section{Conclusions}

The emerging development of consumer technology provides unprecedented opportunities to evaluate the use of additional digital biomarkers to improve risk management strategies for population health and for precision health at the level of an individual. Paired with access to large population studies, such as the AoURP [30] that collects health questionnaires, electronic health records, physical measurements, biospecimens, and digital health technology data, we can rapidly test emerging digital health measures for their potential to advance precision medicine. The heart snapshot software is freely available with all validation data and analysis code [37].

\section{Acknowledgments}

The authors would like to acknowledge Steve Steinhubl, Shannon Young, Nathaniel Brown, Joshua Liu, Erin Mounts, Stockard Simon, and Woody MacDuffie for their contributions to this work. Data are made available through Synapse (DOI: 10.7303/syn22107959).

\section{Authors' Contributions}

DEW and LO wrote the first draft of the paper. LO, MK, and JG developed the study and protocol, and MT developed algorithms for heart rate measurements. LO and MT performed the analyses. MH, DW, and JG recruited the participants and performed all measurements in the laboratory. MK and DEW oversaw the design and development of the heart snapshot apps. EA, VK, MVM, EM, JO, and LM helped identify the protocols for generalization, provided expert input, and edited the paper together with MK, LO, JG, DW, MT, MH, and DEW. LO and MK contributed equally to this study

\section{Conflicts of Interest}

EA is a founder and advisor for Personalis and Deep Cell and collaborates scientifically with Apple Inc. MVM is currently employed at Google.

\section{Multimedia Appendix 1}

Self-guided instructions and screen workflow for performing the heart snapshot $\mathrm{VO}<\mathrm{sub}>2</$ sub $>$ max estimate. [PNG File, 223 KB-Multimedia Appendix 1] 


\section{Multimedia Appendix 2}

Demographic data for the maximal oxygen consumption validation study.

[XLSX File (Microsoft Excel File), 29 KB-Multimedia Appendix 2]

\section{References}

1. Wilson PW, D'Agostino RB, Levy D, Belanger AM, Silbershatz H, Kannel WB. Prediction of coronary heart disease using risk factor categories. Circulation 1998 May 12;97(18):1837-1847. [doi: 10.1161/01.cir.97.18.1837] [Medline: 9603539]

2. Ridker PM, Buring JE, Rifai N, Cook NR. Development and validation of improved algorithms for the assessment of global cardiovascular risk in women: the Reynolds Risk Score. J Am Med Assoc 2007 Feb 14;297(6):611-619. [doi: 10.1001/jama.297.6.611] [Medline: 17299196]

3. Hippisley-Cox J, Coupland C, Brindle P. Development and validation of QRISK3 risk prediction algorithms to estimate future risk of cardiovascular disease: prospective cohort study. Br Med J 2017 May 23;357:j2099 [FREE Full text] [doi: 10.1136/bmj.j2099] [Medline: 28536104]

4. Laukkanen JA, Rauramaa R, Salonen JT, Kurl S. The predictive value of cardiorespiratory fitness combined with coronary risk evaluation and the risk of cardiovascular and all-cause death. J Intern Med 2007 Aug;262(2):263-272 [FREE Full text] [doi: 10.1111/j.1365-2796.2007.01807.x] [Medline: 17645594]

5. Mandsager K, Harb S, Cremer P, Phelan D, Nissen SE, Jaber W. Association of cardiorespiratory fitness with long-term mortality among adults undergoing exercise treadmill testing. JAMA Netw Open 2018 Oct 05;1(6):e183605 [FREE Full text] [doi: 10.1001/jamanetworkopen.2018.3605] [Medline: 30646252]

6. Ross R, Blair SN, Arena R, Church TS, Després J, Franklin BA, American Heart Association Physical Activity Committee of the Council on LifestyleCardiometabolic Health, Council on Clinical Cardiology, Council on EpidemiologyPrevention, Council on CardiovascularStroke Nursing, Council on Functional GenomicsTranslational Biology, Stroke Council. Importance of assessing cardiorespiratory fitness in clinical practice: a case for fitness as a clinical vital sign: a scientific statement from the American Heart Association. Circulation 2016 Dec 13;134(24):653-699. [doi: 10.1161/CIR.0000000000000461] [Medline: 27881567]

7. Guo Y, Bian J, Li Q, Leavitt T, Rosenberg EI, Buford TW, et al. A 3-minute test of cardiorespiratory fitness for use in primary care clinics. PLoS One 2018;13(7):e0201598 [FREE Full text] [doi: 10.1371/journal.pone.0201598] [Medline: $\underline{30059539]}$

8. Rippe JM, editor. Guidelines for exercise testing and prescription (ACSM). In: Encyclopedia of Lifestyle Medicine and Health. Thousand Oaks, California, United States: SAGE Publications Inc; 2012:1-1296.

9. Balady GJ, Arena R, Sietsema K, Myers J, Coke L, Fletcher GF, et al. Clinician's guide to cardiopulmonary exercise testing in adults. Circulation 2010 Jul 13;122(2):191-225 [FREE Full text] [doi: 10.1161/cir.0b013e3181e52e69]

10. McDavid A, Crane PK, Newton KM, Crosslin DR, McCormick W, Weston N, et al. Enhancing the power of genetic association studies through the use of silver standard cases derived from electronic medical records. PLoS One 2013;8(6):e63481 [FREE Full text] [doi: 10.1371/journal.pone.0063481] [Medline: 23762230]

11. Noonan V, Dean E. Submaximal exercise testing: clinical application and interpretation. Phys Ther 2000 Aug;80(8):782-807. [Medline: $\underline{10911416}$ ]

12. Bennett H, Parfitt G, Davison K, Eston R. Validity of submaximal step tests to estimate maximal oxygen uptake in healthy adults. Sports Med 2016 May;46(5):737-750. [doi: 10.1007/s40279-015-0445-1] [Medline: 26670455]

13. Muntaner-Mas A, Martinez-Nicolas A, Lavie CJ, Blair SN, Ross R, Arena R, et al. A systematic review of fitness apps and their potential clinical and sports utility for objective and remote assessment of cardiorespiratory fitness. Sports Med 2019 Apr;49(4):587-600 [FREE Full text] [doi: 10.1007/s40279-019-01084-y] [Medline: 30825094]

14. Cooper KH. A means of assessing maximal oxygen intake. Correlation between field and treadmill testing. J Am Med Assoc 1968 Jan 15;203(3):201-204. [Medline: 5694044]

15. Hughes $\mathrm{AD}$, Chaturvedi N. Estimation of maximal oxygen consumption and heart rate recovery using the tecumseh sub-maximal step test and their relationship to cardiovascular risk factors. Artery Res 2017 Jun;18:29-35 [FREE Full text] [doi: 10.1016/j.artres.2017.02.005] [Medline: 28546848]

16. Goodman JM, Thomas SG, Burr J. Evidence-based risk assessment and recommendations for exercise testing and physical activity clearance in apparently healthy individuals. Appl Physiol Nutr Metab 2011 Jul;36 Suppl 1:14-32. [doi: 10.1139/h11-048] [Medline: 21800940]

17. Borg GA. Psychophysical bases of perceived exertion. Med Sci Sports Exerc 1982;14(5):377-381. [Medline: 7154893 ]

18. Montoye HJ, Block W, Keller JB, Willis PW. Fitness, fatness, and serum cholesterol: an epidemiological study of an entire community. Res Q Am Allian Health Phy Edu Recreat 2013 Mar 17;47(3):400-408 [FREE Full text] [doi: 10.1080/10671315.1976.10615390]

19. Milligan G. Fitness standards for the Maritime and Coastguard Agency and the oil and gas industry. University of Portsmouth. 2013. URL: https://pdfs.semanticscholar.org/739c/d165a84c7d7146a745580d0ad8e593f4dd3c.pdf [accessed 2021-05-13] 
20. Eilers S, Bach DQ, Gaber R, Blatt H, Guevara Y, Nitsche K, et al. Accuracy of self-report in assessing Fitzpatrick skin phototypes I through VI. JAMA Dermatol 2013 Nov;149(11):1289-1294. [doi: 10.1001/jamadermatol.2013.6101] [Medline: 24048361]

21. Reeder AI, Hammond VA, Gray AR. Questionnaire items to assess skin color and erythemal sensitivity: reliability, validity, and "the dark shift". Cancer Epidemiol Biomarkers Prev 2010 May;19(5):1167-1173 [FREE Full text] [doi: 10.1158/1055-9965.EPI-09-1300] [Medline: 20447914]

22. Bino SD, Bernerd F. Variations in skin colour and the biological consequences of ultraviolet radiation exposure. Br $\mathrm{J}$ Dermatol 2013 Oct;169 Suppl 3:33-40. [doi: 10.1111/bjd.12529] [Medline: 24098899]

23. Bland JM, Altman D. Statistical methods for assessing agreement between two methods of clinical measurement. Lancet 1986 Feb;327(8476):307-310. [doi: 10.1016/S0140-6736(86)90837-8]

24. Lin LI. A concordance correlation coefficient to evaluate reproducibility. Biometrics 1989 Mar;45(1):255. [doi: 10.2307/2532051]

25. CardiorespiratoryFitness-Android. Sage-Bionetworks. URL: https://github.com/Sage-Bionetworks/ CardiorespiratoryFitness-Android [accessed 2020-11-24]

26. CardiorespiratoryFitness-iOS. Sage-Bionetworks. URL: https://github.com/Sage-Bionetworks/CardiorespiratoryFitness-iOS [accessed 2020-11-24]

27. Milligan G, House J, Tipton M, Hildenbrand B, Maeso M. A recommended fitness standard for the oil and gas industry. In: Proceedings of the European HSE Conference and Exhibition. 2013 Presented at: European HSE Conference and Exhibition; April 2013; London, United Kingdom. [doi: 10.2118/164960-MS]

28. Bandyopadhyay A. Validity of Cooper's 12-minute run test for estimation of maximum oxygen uptake in male university students. Biol Sport 2015 Mar;32(1):59-63 [FREE Full text] [doi: 10.5604/20831862.1127283] [Medline: 25729151]

29. Weisgerber M, Danduran M, Meurer J, Hartmann K, Berger S, Flores G. Evaluation of Cooper 12-minute walk/run test as a marker of cardiorespiratory fitness in young urban children with persistent asthma. Clin J Sport Med 2009 Jul;19(4):300-305. [doi: 10.1097/JSM.0b013e3181b2077a] [Medline: 19638824]

30. All of Us Research Program Investigators, Denny JC, Rutter JL, Goldstein DB, Philippakis A, Smoller JW, et al. The "All of Us" research program. N Engl J Med 2019 Aug 15;381(7):668-676. [doi: 10.1056/NEJMsr1809937] [Medline: 31412182]

31. Shcherbina A, Mattsson CM, Waggott D, Salisbury H, Christle JW, Hastie T, et al. Accuracy in wrist-worn, sensor-based measurements of heart rate and energy expenditure in a diverse cohort. J Pers Med 2017 May 24;7(2):3 [FREE Full text] [doi: 10.3390/jpm7020003] [Medline: 28538708]

32. Bent B, Goldstein BA, Kibbe WA, Dunn JP. Investigating sources of inaccuracy in wearable optical heart rate sensors. NPJ Digit Med 2020;3:18 [FREE Full text] [doi: 10.1038/s41746-020-0226-6] [Medline: 32047863]

33. Fitzpatrick TB. The validity and practicality of sun-reactive skin types I through VI. Arch Dermatol 1988 Jun;124(6):869-871. [doi: 10.1001/archderm.124.6.869] [Medline: $\underline{3377516}$ ]

34. Ridder BD, Van Rompaey B, Kampen JK, Haine S, Dilles T. Smartphone apps using photoplethysmography for heart rate monitoring: meta-analysis. JMIR Cardio 2018 Feb 27;2(1):e4 [FREE Full text] [doi: 10.2196/cardio.8802] [Medline: $\underline{31758768]}$

35. Klepin K, Wing D, Higgins M, Nichols J, Godino JG. Validity of cardiorespiratory fitness measured with Fitbit compared to V·O2max. Med Sci Sports Exerc 2019 Nov;51(11):2251-2256 [FREE Full text] [doi: 10.1249/MSS.0000000000002041] [Medline: $\underline{31107835}$ ]

36. Avram R, Tison GH, Aschbacher K, Kuhar P, Vittinghoff E, Butzner M, et al. Real-world heart rate norms in the Health eHeart study. NPJ Digit Med 2019;2:58 [FREE Full text] [doi: 10.1038/s41746-019-0134-9] [Medline: $\underline{31304404]}$

37. CRF validation analysis. Sage-Bionetworks. URL: https://github.com/Sage-Bionetworks/CRF validation analysis [accessed 2020-11-06]

\author{
Abbreviations \\ 3-MST: 3-minute step test \\ 12-MRT: 12-minute run test \\ ACF: autocorrelation function \\ AoURP: All of Us Research Program \\ EPARC: Exercise and Physical Activity Resource Center \\ UCSD: University of California, San Diego \\ $\mathbf{V O}_{2}$ max: maximal oxygen consumption
}


Edited by L Buis; submitted 24.11.20; peer-reviewed by M Altini, CP Lau; comments to author 11.01.21; revised version received 04.02.21; accepted 12.04.21; published 04.06.21

Please cite as:

Webster DE, Tummalacherla M, Higgins M, Wing D, Ashley E, Kelly VE, McConnell MV, Muse ED, Olgin JE, Mangravite LM, Godino J, Kellen MR, Omberg L

Smartphone-Based VO2max Measurement With Heart Snapshot in Clinical and Real-world Settings With a Diverse Population: Validation Study

JMIR Mhealth Uhealth 2021;9(6):e26006

URL: https://mhealth.jmir.org/2021/6/e26006

doi: $10.2196 / 26006$

PMID:

(CDan E Webster, Meghasyam Tummalacherla, Michael Higgins, David Wing, Euan Ashley, Valerie E Kelly, Michael V McConnell, Evan D Muse, Jeffrey E Olgin, Lara M Mangravite, Job Godino, Michael R Kellen, Larsson Omberg. Originally published in JMIR mHealth and uHealth (https://mhealth.jmir.org), 04.06.2021. This is an open-access article distributed under the terms of the Creative Commons Attribution License (https://creativecommons.org/licenses/by/4.0/), which permits unrestricted use, distribution, and reproduction in any medium, provided the original work, first published in JMIR mHealth and uHealth, is properly cited. The complete bibliographic information, a link to the original publication on https://mhealth.jmir.org/, as well as this copyright and license information must be included. 